\title{
Diagnostic value of a panel of tumor markers as a part of a diagnostic work-up for ascites of unknown etiology
}

\author{
Soha Saoud Abdelmoniem ${ }^{1 *}$, Eman Mosad Zaki ${ }^{2}$, Hala Mostafa Imam ${ }^{3}$, Hosny Badrawy ${ }^{2}$, Sanaa Ali ${ }^{4}$, \\ Doaa Wadeeh Maximous ${ }^{5}$ \\ ${ }^{1}$ Tropical Medicine Department, Faculty of Medicine, Assiut University, Assiut, Egypt \\ ${ }^{2}$ Clinical Pathology Department, Molecular Biology Lab, South Egypt Cancer Institute, Assiut University, Assiut, Egypt \\ ${ }^{3}$ Department of Internal Medicine, Faculty of Medicine, Assiut University, Assiut, Egypt \\ ${ }^{4}$ Clinical Pathology Department, Faculty of Medicine, Quina University, Quina, Egypt \\ ${ }^{5}$ Surgical Oncology Department, South Egypt Cancer Institute, Assiut University, Assiut, Egypt \\ Email: sosaoud@hotmail.com
}

Received 11 March 2012; revised 12 April 2012; accepted 19 April 2012

\section{ABSTRACT}

Background \& Objectives: Data regarding tumor marker usefulness in diagnosing ascites of unknown etiology and determining its malignant nature are conflicting. We aim to assess the diagnostic value of ascitic and serum tumor markers in ascites of unknown etiology and to evaluate their usefulness besides other laboratory tests in a diagnostic work-up in those patients. Design \& Setting: A prospective case-control study conducted at Assiut University hospital and oncology institute. Patients \& Methods: Three groups were included; Group I: 41 patients with ascites of unknown etiology Group II: 7 Patients with TB ascites and Group III:14 patients with cirrhotic ascites. We assessed the CEA, CEA mRNA, CA15-3, CA19.9, CA125, AFP and PSA in serum and ascetic fluid. A diagnostic workup for group I included: IPD test, ultrasound, CT, ascetic fluid cytology, SAAG, Laparotomy and biopsy. Results: Ascetic fluid and serum levels of CA15-3 and CA125 were significantly increased in group $I$ and were significantly increased in histopathologically proved malignant ascites compared to TB and cirrhotic ascites. In group I, CA125 was significantly higher in ascites than serum. With the exception of PSA, all tumor markers significantly correlated in serum and ascetic fluid. No significant difference in the level of ascetic CEA messenger RNA was detected between the 3 groups. Cytology had $53 \%$ sensitivity, $94 \%$ specificity and CA 125 \& CA15-3 had 81\% sensitivity and $75 \%$ specificity in detection of malignant ascites respectively. Laparotomy and Biopsy: Diagnosed malignnancy in $53.3 \%$ and TB in $\mathbf{1 3 . 3 \%}$. Conclusions: A diagnostic work-up including SAAG, tumor markers in the serum and ascetic fluid may help in adjunct with

\footnotetext{
"Corresponding author.
}

ascetic fluid cytology, laparotomy and biopsy, imaging and other laboratory tests in diagnosing ascites of unknown etiology.

Keywords: Tumor Markers; Ascites of Unknown Etiology

\section{INTRODUCTION}

The etiology of ascites can be recognized in most of patients by clinical examinations and conventional laboratory tests, however, occasionally it remains undetermined without further investigation. Ascites of unknown origin can be defined as the ascites in which the etiology cannot be determined after conventional laboratory examinations (including cell count, albumin level, total protein level, Gram stain, and culture) and further imaging investigations (including ultrasound and CT scan) [1].

A variety of biochemical markers were introduced to distinguish malignant and nonmalignant ascites [2]. Such markers are detected in the serum, and may also be analyzed in ascetic fluid to increase diagnostic performance $[3,4]$. Examples include alpha fetoprotein (AFP), carcinoembryonic antigen (CEA), cancer antigen CA19-9, CA15-3, and $\mathrm{CA} 125$ [5]. However, previous reports about their diagnostic ability are conflicting. The discrepancy in results can be attributed to the heterogeneity of tumor types, different assay methodologies and cutoff values [2,3].

Reverse transcriptase-polymerase chain reaction (RTPCR) has been utilized to detect living micrometastases of cancer cells in the lymph node, ascites or serum. This can be done by quantification of tumor markers mRNA using real-time PCR to detect living cancer cells in the serum and ascetic fluid [6].

Data regarding tumor marker usefulness in diagnosing unknown ascites and determining the malignant nature of 
ascites are conflicting and insufficient. Consequently the aim of this study was to assess the diagnostic value of a group of tumor markers levels in serum and ascetic fluid along with other known biochemical tests and imaging techniques in patients with ascites of unknown etiology.

\section{PATIENTS \& METHODS}

\subsection{Patients}

This study was conducted at Assiut University hospital and Assiut oncology institute. Patients admitted with primary diagnosis of ascites from January, 2007 to January, 2009 were prospectively included in this study. Patients were categorized into3 groups:

- Group I: 41 patients with ascites of unknown etiology.

- Group II: 7 patients with TB ascites.

- Group III: 14 patients with cirrhotic ascites.

Detailed history and clinical examinations were obtained in all patients.

\subsection{Methods}

\subsubsection{Diagnostic Work-Up in Group I}

The diagnostic work-up for group I included:

- Search for tuberculous etiology with a chest x-ray, staining for acid fast bacillus in sputum, urine, gastric aspiration products, and ascetic fluid by Zeil-Nelson stain, and a tuberculin intradermal reaction.

- Gynecology examination together with pelvic ultrasound (US) in women.

- Liver function tests, Prothrombin time, blood urea, serum creatinine, urine analysis, $24 \mathrm{hr}$. urine protein, $\mathrm{T} 3, \mathrm{~T} 4, \mathrm{TSH}$, and serum cholesterol.

- Upper endoscopy.

- Ascetic fluid study: included ascetic fluid protein, cells, SAAG, conventional cytological examination of ascetic fluid was performed using haematoxylin and eosin, and Giemsa stains. Analyses were repeated three times before malignancy was excluded.

- ANA, LE cells, c-reactive protein and amyloid1 test.

- An abdominal computed tomography (CT) scan, echocardiography, abdominal conventional US and Doppler US.

- An exploratory laparotomy was performed in undiagnosed patients to evaluate the gross pathology. Peritoneal biopsies and liver biopsy were obtained when possible.

\subsubsection{Tumor Markers Assessment}

Done in the three groups both in the serum and ascetic fluid:

- Blood samples were obtained and serum was separated aliquot in small volumes and stored at $-20^{\circ} \mathrm{C}$ until use.

- Ascetic fluid samples obtained were collected in tubes containing ethylenediamine tetra-acetic acid, centri- fuged at $3000 \mathrm{~g}$, and stored at $-70^{\circ} \mathrm{C}$ until assayed.

- Carcinoembryonic antigen (CEA), CEA mRNA, cancer antigen CA125, CA19-9, CA15-3, alpha-feto-protein (AFP) and PSA were measured in serum and peritoneal fluid according to instruction manual using an electrochemiluminescence immunoassay on Roche Elecsys 2010 analyzers (Roche Diagnostics; Mannheim, Germany).

- All tumor markers assays were performed at South Egypt Cancer Institute, Assiut University. Serum CEA, CA125, CA15-3, CA19-9, PSA and alpha fetoprotein levels $<2.5 \mathrm{ng} / \mathrm{ml}, 35 \mathrm{U} / \mathrm{ml},<3 \mathrm{U} / \mathrm{ml},<33 \mathrm{U} / \mathrm{ml}$, up to $4 \mathrm{ng} / \mathrm{ml}$ and $<10 \mu \mathrm{g} / \mathrm{l}$, respectively, are adopted as the upper limit.

\subsubsection{Ascetic Fluid CEA mRNA Assessment}

Total RNA was extracted from ascetic fluids in the three groups by Simply P Total RNA Extraction kit (BioFlux). PCR was done by illustra ${ }^{\text {TM }}$ Ready-To-Go RT-PCR Beads (GE Healthcare). Ready-To-Go RT-PCR Beads utilize Moloney Murine Leukemia Viurs (M-MULV) reverse transcriptase and Taq DNA polymerase to generate PCR product from an RNA template. Each bead is optimized to allow the first-strand cDNA synthesis and PCR reaction to proceed sequentially as a single-tube, singlestep reaction.

\subsubsection{Statistical Analysis}

Data were expressed as median and interquartile ranges. Statistical analysis was performed using the statistical package (SPSS) version 16.0; Chicago, IL. The level of $\mathrm{P}<0.05$ was considered the cut-off value for significance.

Since, the tumor marker data in serum and ascetic fluid were skewed. Data transformation can convert a skewed distribution into a symmetrical form and logarithmic transformation compresses high values together and stretches smaller values apart and can also be used to stabilize the variation of a sample. Logarithmic transformation was, therefore, applied to the present data and the means and SDs were calculated. Comparisons between the 3 groups used Kruskal-Wallis test. Student's t-test for two independent samples was used to compare the concentration means of the tumor markers. Positive predictive value (PPV) and negative predictive value (NPV) were also calculated.

\subsubsection{Ethical Consideration}

The study protocol has been approved by the ethical committee of research of Faculty of medicine, Assiut University, and informed consent has been taken before conducting the study.

\section{RESULTS}

A total of 62 patients (47 males, 15 females) with ascites 
were enrolled into the study (41 patients with ascites of unknown etiology (group I), 7 patients with TB ascites (group II), and 14 patients with cirrhotic ascites (group III)). Comparison of demographic data, hematological, and biochemical tests between the 3 groups were presented in Table 1.

\subsection{Results of the Diagnostic Work-Up in Group I}

Thirty patients in group I had low-gradient SAAG, nine of those showed positive cytology for malignancy, 27 of them showed abdominal masses by CT scan and 15 of those were positive for CA125 and CA15-3 in both the serum and ascetic fluid. Those patients were suitable for laparotomy and biopsy; of those biopsy revealed 16 patients with malignancy, 4 patients with TB peritonitis, one patient with pancreatitis and 9 patients no abnormalities could be detected by laparotomy and no biopsy was taken. Further detailed history and laboratory tests for the latter group diagnosed them as nephrotic syndrome in $3(33.3 \%)$ patients, Familial Mediterranean fever in 2 (22.2\%) patients, hypothyroidism in $3(33.3 \%)$ patients, and the remaining $1(11.1 \%)$ patients the etiology was undetermined.

Eleven patients of group I showed high-gradient SAAG, negative cytology for malignancy, no abdominal masses by $\mathrm{CT}$ and positive CA 125 in both serum and ascetic fluid in $2(18.2 \%)$ patients. Three $(27.5 \%)$ patients dropped from the study for social reasons. Further laboratory tests, echocardiography, upper endoscopy and Doppler US, 1 (9.1\%) patients was diagnosed as Budd chiari syndrome, $2(18.2 \%)$ patients were diagnosed as liver cirrhosis, 2 $(18.2 \%)$ patients were diagnosed as having mild constrictive and dilated cardiomyopathy, $2(18.2 \%)$ patients were diagnosed as SLE, and one (9.1\%) patient remained undiagnosed.

\subsection{Ascetic Fluid Study Results}

SAAG was $<1.1$ (non portal hypertensive ascites) in $30 / 41(73.17 \%)$ patients in group I, while in group II SAAG was $<1.1$ in $4 / 7$ (57.1\%). In group III SAAG was $\geq 1.1$ (portal hypertensive ascites) in 10/14 (71.42\%). The PPV of SAAG for the diagnosis of non-portal hyperten- sive ascites was $89.47 \%$, while, NPV was $41.66 \%$. As- cetic fluid cytology was negative for malignant cells in group II and III, while, malignant cells was found in $9 / 41(22 \%)$ in group I.

\subsection{Tumor Markers Results}

CEA, CA19.9, AFP, and PAS were negative in both serum and ascetic fluid in the three groups while CA125 and CA15-3 were elevated in the serum and ascetic fluid in the three groups. Comparison between the tumor markers levels in the serum and ascetic fluid between the three groups was presented in Table 2 . The only statistical significant difference in tumor markers values between the 3 groups was in ascetic CA15-3 and CA125 that were higher in group I compared to group II and III $(\mathrm{P}<0.05)$.

When comparing serum and ascetic fluid tumor markers levels in group I after Logarithmic transformation of values Table 3, no statistical significance difference was found between most of tumors markers except for CA125 that was higher in ascites than in serum $(\mathrm{P}<0.001)$.We also studied the correlations between tumor markers in ascetic fluid and serum among patients in group I to determine whether the ascetic fluid analysis had any diagnostic advantage over its serum value. Serum and ascetic tumor markers (CEA, CA125, CA15-3, AFP, CA19-9) statistically significantly correlated positively with each other $(\mathrm{P}<0.001)$, with the exception of PSA which negatively correlated and did not reach statistical significance.

Table 1. Demographic, hematological and biochemical data in the 3 studied groups (median and quartiles).

\begin{tabular}{cccc}
\hline & $\begin{array}{c}\text { Group I }(\mathrm{N}=41) \\
\text { Ascites of unknown etiology } \\
\text { Median (Q1-Q3) }\end{array}$ & $\begin{array}{c}\text { Group II }(\mathrm{N}=7) \\
\text { TB ascites } \\
\text { Median (Q1-Q3) }\end{array}$ & $\begin{array}{c}\text { Group III }(\mathrm{N}=14) \\
\text { Cirrhotic ascites } \\
\text { Median }(\mathrm{Q} 1-\mathrm{Q} 3)\end{array}$ \\
\cline { 2 - 4 } Age (years) & $49(44-54)$ & $50(40-51)$ & $50(44-56)$ \\
Sex (M:F) & $33: 8$ & $4: 3$ & $10: 4$ \\
WBCs/uL & $7(6-9)$ & $8(7-16)$ & $6.5(3.9-9.2)$ \\
RBCs/uL & $3.4(2.8-4)$ & $3(2-4)$ & $3.2(2.8-4)$ \\
Platelet/uL & $238(169-340)$ & $327(160-342)$ & $100(85-173)^{* *}$ \\
Hemoglobin (g/dl) & $10.3(9.4-12.4)$ & $10(9.9-11)$ & $10(9.7-10.5)$ \\
Total bilirubin (mg/dl) & $9.9(5.6-16)$ & $4(3.1-7)$ & $20(13.3-29.3)$ \\
AST (IU/l) & $26(20-36.5)$ & $33(23-39)$ & $52(37.3-64)^{*}$ \\
ALT (IU/l) & $17(11.4-29.5)$ & $20(14-31)$ & $39(27.5-66)^{*}$ \\
Total protein (g/l) & $59(44.5-67)$ & $66(56-69)$ & $47(28-60.8)^{*}$ \\
Albumin (g/l) & $33(29.5-36)$ & $33(25-34)$ & $23(19.8-27)^{*}$ \\
ALP (IU/l) & $106(80-129)$ & $101(77-132)$ & $101(73-136)$ \\
SAAG & $0.7(0.5-1.2)$ & $0.80(0.6-1.8)$ & $1.5(0.77-1.7)$ \\
\hline
\end{tabular}

ALT: alanine aminotransferase; AST: aspartate aminotransferase; ALP: alkaline phosphatase; SAAG: serum ascetic albumin gradient; Kruskal-Wallis test ${ }^{*} \mathrm{P}$ value $<0.05,{ }^{* *} \mathrm{P}$ value $<0.001$ 
Table 2. Comparison of the tumor markers in the serum and ascetic fluid between the 3 studied groups (median and quartiles).

\begin{tabular}{cccc}
\hline Tumour marker & $\begin{array}{c}\text { Group I }(\mathrm{N}=41) \\
\text { Ascites of unknown etiology } \\
\text { Median }(\mathrm{Q} 1-\mathrm{Q})\end{array}$ & $\begin{array}{c}\text { Group II }(\mathrm{N}=7) \\
\text { TB ascites } \\
\text { Median }(\mathrm{Q} 1-\mathrm{Q} 3)\end{array}$ & $\begin{array}{c}\text { Group III }(\mathrm{N}=14) \\
\text { Cirrhotic ascites } \\
\text { Median }(\mathrm{Q} 1-\mathrm{Q} 3)\end{array}$ \\
\hline Ascetic CEA $(\mathrm{ng} / \mathrm{ml})$ & $1.58(0.9-1.9)$ & $1.86(0.7-1.9)$ & $0.9(0.4-1.9)$ \\
Serum CEA $(\mathrm{ng} / \mathrm{ml})$ & $1.8(1.4-2.7)$ & $1.36(0.6-2.5)$ & $1.9(1.4-4.5)$ \\
Ascetic CEA messenger RNA & $1(1-1)$ & $1(1-1)$ & $1(1-1)$ \\
Ascetic CA19.9 $(\mathrm{U} / \mathrm{ml})$ & $2.9(0.6-8.3)$ & $2.9(0.6-8)$ & $3.7(0.5-8.5)$ \\
Serum CA19.9 $(\mathrm{U} / \mathrm{ml})$ & $8.3(0.9-19.4)$ & $7.3(0.9-18.5)$ & $8.2(3.5-19.7)$ \\
Ascetic CA15.3 $(\mathrm{U} / \mathrm{ml})$ & $15(12-33)^{*}$ & $9(6-11.7)$ & $10(5.5-20)$ \\
Serum CA15.3 $(\mathrm{U} / \mathrm{ml})$ & $15(5-34)$ & $12.5(4.2-34)$ & $12(6-32)$ \\
Ascetic AFP $(\mathrm{ng} / \mathrm{ml})$ & $1.6(1.3-4)$ & $0.6(1-3.2)$ & $1.3(1-3.2)$ \\
Serum AFP $(\mathrm{ng} / \mathrm{ml})$ & $2.8(0.8-5.7)$ & $2.8(1.1-5.7)$ & $2(1-5.3)$ \\
Ascetic CA125 $(\mathrm{U} / \mathrm{ml})$ & $774(367.5-1666)^{*}$ & $509.5(343.2-900)$ & $500(211-827)$ \\
Serum CA125 $(\mathrm{U} / \mathrm{ml})$ & $390(164-456.5)$ & $291.5(164-435)$ & $176(185.5-396)$ \\
Ascetic PSA $(\mathrm{ng} / \mathrm{ml})$ & $0.65(0.4-1.1)$ & $0.8(0.4-1.2)$ & $0.90(0.6-1.2)$ \\
Serum PSA $(\mathrm{ng} / \mathrm{ml})$ & $1(0.6-1.0)$ & $1(0.9-1.2)$ & $1(0.9-1.2)$ \\
\hline
\end{tabular}

CA: cancer antigen; CEA: carciniemberyonic antigen; AFP: alpha-feto protein; PSA: prostate-specific antigen; By Kruskal-Wallis test " $\mathrm{P}$ value $<0.05$

Table 3. Comparison between serum and ascetic fluid levels of tumor marker (logarithmically transformed, mean(SD) in patients in group I (ascites of unknown etiology).

\begin{tabular}{ccc}
\hline Tumour marker & Ascitic fluid & Serum \\
\hline CEA $(\mathrm{ng} / \mathrm{ml})$ & $0.2(0.5)$ & $0.3(0.3)$ \\
CA $15.3(\mathrm{U} / \mathrm{ml})$ & $1.1(0.6)$ & $1.1(0.6)$ \\
AFP $(\mathrm{ng} / \mathrm{ml})$ & $0.3(0.5)$ & $0.6(0.7)$ \\
CA125 $(\mathrm{U} / \mathrm{ml})$ & $\mathbf{2 . 3 ( 0 . 8 )}$ & $\mathbf{0 . 2 ( 0 . 4 )}$ \\
CA19.9 $(\mathrm{U} / \mathrm{ml})$ & $0.5(0.9)$ & $0.7(0.9)$ \\
PAS $(\mathrm{ng} / \mathrm{ml})$ & $0.9(0.5)$ & $1.5(0.7)$ \\
\hline
\end{tabular}

CA: cancer antigen; CEA: carciniemberyonic antigen; AFP: alpha-feto protein; PSA: prostate-specific antigen; ${ }^{* *} \mathrm{P}$ value $<0.001$ by paired T test.

When we Compared between tumor markers values in the serum and ascetic fluid among patients diagnosed by laparotomy and histopathologically as having malignant ascites, TB and group III, results showed statistical significant increase in both serum and ascetic fluid levels of CA15-3 and CA125 in malignant ascites than in those with TB ascites including group II or group III (cirrhotic ascites) $(\mathrm{P}<0.05)$ Table 4.

No statistical significant difference in the level of ascetic CEA messenger RNA was detected between the 3 groups.

\subsection{Sensitivity and Specificity of Tumor Markers in Group I}

Among thirty patients in group I who underwent laparotomy and biopsy, CA125 and CA15-3 were positive in the serum and ascetic fluid in $15 / 30(50 \%)$ patients, 13 $(86.7 \%)$ of them were proven to have malignancy $(7 / 13$ had ovarian cancer, 2/13 had colon cancer, 2/13 had pancreatic cancer and 2/13 with HCC) by histopathology and $2(13.3 \%)$ were negative for malignancy. CA125 and CA15-3 were negative in $15 / 30$ patients, $3(20 \%)$ of them were positive for malignancy ( $1 / 3$ had peritoneal carcinomatosis, $1 / 3$ had metastatic carcinoma in the liver and $1 / 3$ had hepatic heamangioma) and 12 (80\%) were negative for malignancy. On the other hand, CA125 was positive in two patients out of eleven patients who did not undergo laparotomy and were diagnosed as liver cirrhosis. Therefore, the sensitivity of tumor markers is $81 \%$ and its specificity is $75 \%$.

\subsection{Imaging Study Results}

US of the liver in group I showed that $46 \%$ had normal liver, $12 \%$ had focal lesions in the liver, $12 \%$ had fatty liver, and $30 \%$ had hepatomegaly of unexplained etiology, while $34 \%$ patients had mild splenomegaly. In group III, $2 / 14(14.5 \%)$ patients had hepatic focal lesions diagnosed as HCC and in group II all patients had ascites with internal echoes and one patient had fatty liver. CT scan showed abnormalities in $27 / 41(66 \%)$ patients in group I; ovarian masses, colorectal lesions, hepatic focal lesions, pancreatic lesions in 10/41 (24.4\%), 2/41 (4.9\%), $12 / 41(29.3 \%), 3 / 41(7.3 \%)$ and $14 / 41(34.1 \%)$ and no abnormalities in $14 / 41$ (34\%) patients. In group III hepatic focal lesions were detected in 4/14 (28.6\%) patients. No abnormality except for ascites in all patients and ovarian mass in one patient could be detected in group II.

\subsection{Laparotomy and Biopsy Results}

Thirty patients in group I were candidates for exploratory laparotomy. Laparotomy and biopsy results showed that $16 / 30(53.3 \%)$ patients were diagnosed by histopathology as malignant ascites. Malignancy associated was ovarian carcinoma, colorectal carcinoma, pancreatic carcinoma, HCC, hepatic heamangioma, metastatic carcinoma in the liver and peritoneal carcinomatosis were diagnosed in 
Table 4. Comparison between levels of tumor markers in serum and ascetic fluid among patients diagnosed histopathologically as having malignant, TB and cirrhotic ascites.

\begin{tabular}{cccc}
\hline \multirow{2}{*}{ Tumour marker } & Malignant ascites & TB ascites & Cirrhotic ascites \\
& $\mathrm{N}=16$ & $\mathrm{~N}=11(7+4)$ & $\mathrm{N}=14$ \\
& Median $(\mathrm{Q} 1-\mathrm{Q} 3)$ & Median $(\mathrm{Q} 1-\mathrm{Q} 3)$ & Median $(\mathrm{Q} 1-\mathrm{Q})$ \\
\hline Ascetic CEA & $1.6(0.9-2)$ & $1.9(0.6-1.9)$ & $0.9(0.3-1.9)$ \\
Serum CEA & $1.9(1.3-2.8)$ & $1.3(0.5-2.3)$ & $1.9(1.4-4.4)$ \\
CEA messenger RNA & $1(1-1)$ & $1(1-1)$ & $1(1-1)$ \\
Ascetic CA19.9 & $2.9(0.6-8.3)$ & $2.9(0.6-8)$ & $3.7(0.5-8.5)$ \\
Serum CA19.9 & $8.5(1.7-20.6)$ & $6.2(0.7-1.42)$ & $8.2(3.5-19.7)$ \\
Ascetic CA15.3 & $25(17-39)^{*}$ & $7.5(5-12.9)$ & $10(5.5-20)$ \\
Serum CA15.3 & $19(5.5-36)^{*}$ & $10.5(5.3-30.2)$ & $12(6-32)$ \\
Ascetic AFP & $1.7(1.5-4)$ & $0.6(0.4-3.5)$ & $1.3(1-3.2)$ \\
Serum AFP & $2.8(1.2-5.9)$ & $2.7(1.1-5.6)$ & $2(1-5.3)$ \\
Ascetic CA125 & $885(277.5-1666)^{*}$ & $614.5(322.2-885)$ & $500(211-827)$ \\
Serum CA125 & $414(274-475)^{*}$ & $280(152-411)$ & $176(185.5-396)$ \\
Ascetic PSA & $0.6(0.4-1.1)$ & $0.7(0.4-1.1)$ & $0.9(0.6-1.2)$ \\
Serum PSA & $1(0.6-1.1)$ & $1(0.9-1.2)$ & $1(0.9-1.2)$ \\
\hline
\end{tabular}

CA: cancer antigen; CEA: carciniemberyonic antigen; AFP: alpha-feto protein; PSA: prostate-specific antigen; ${ }^{*} \mathrm{P}$ value $<$ 0.05 by Kruskal-Wallis test.

$7 / 16$ (43.8\%), 2/16 (12.5\%), 2/16 (12.5\%), 2/16 (12.5\%) $1 / 16(6.3 \%), 1 / 16(6.3 \%)$ and $1 / 16(6.3 \%)$ respectively. TB ascites was diagnosed in 4/30 (13.3\%) patients as omental mass with or without mesenteric lymph nodes, peritoneal nodules, ovarian mass with or without omental enlargement. One patient/16 (3.3\%) was diagnosed as pancreatitis. Of notice, laparotomy showed no abnormality in $9 / 16$ patients $(30 \%)$.

\subsection{Sensitivity and Specificity of Ascetic Fluid Cytology}

Patients with positive cytology who had undergone laparotomy and biopsy were $9 / 30(30 \%)$. Of those $8 / 30$ $(26.7 \%)$ were proven to be malignant and one was nonmalignant. While patients with negative cytology 21/30 $(67.7 \%), 7(33.3 \%)$ had malignancy and 14 (66.7\%) were non malignant. Therefore, cytology had 53\% sensitivity, 94\% specificity, PPV 89\%, and NPV 70\%.

\section{DISCUSSION}

Tracing the etiology of ascites is still a great challenge. The aim of this study primarily was to assess the diagnostic value of a group of tumor markers in serum and ascetic fluid and detailed ascetic fluid study in patients with ascites of unknown etiology. We included patients who had TB ascites and cirrhotic ascites groups as controls. We also assessed the result of a diagnostic work-up including known laboratory tests in diagnosing patients with ascites of unknown etiology.

We estimated the levels of a group of tumor markers in both the serum and ascetic in the three groups of patients, results revealed that CEA, CA19-9, AFP, and PAS were negative in both serum and ascetic fluid in the three groups while CA125 and CA15-3 were elevated in the serum and ascetic fluid in the three groups. There was also a statistical significance difference regarding the values of ascetic CA15-3 and ascetic CA125 that were higher in group I compared to group II and III.

However, when we considered the malignant ascites patients diagnosed among group I by laparotomy and histopathology, and compared them with TB ascites (both diagnosed by laparotomy and biopsy and group II) and cirrhotic ascites (group III) there was a statistical significant increase in both serum and ascetic fluid levels of CA15-3 and CA125 in malignant ascites compared to the other groups. This was comparable to results by [3] who showed that serum and ascetic CEA, CA 125, and CA19-9 were significantly increased in patients with malignancyrelated ascites compared to those without, however, they were not sensitive parameters in the diagnosis of malignnancy-related ascites.

The study showed that CA125 was positive in two patients with ascites of unknown etiology who were diagnosed as liver cirrhosis and this in concordance with [7] who recorded high levels of CA125 in non-gynecological malignancies and some benign diseases. Moreover, a significant elevation of serum CA125, CA19-9, CA15-3, and CEA were found in the pre-orthotopic liver transplantation (OLT) [6]. Although, these elevations were not associated with tumor diseases in this population. When the levels of serum and ascetic fluid CEA, CA19-9, and CA15-3 were studied in patients with chronic liver disease, spontaneous bacterial peritonitis, malignancy, TB and congestive heart failure and reported that they were not found to be useful in the differential diagnosis of ascites etiology [4].

We have also investigated the correlations between serum and ascetic tumor markers levels. They were significantly correlated positively to each other in group I, 
with the exception of PSA. These results indicate that, analyzing tumor markers in ascetic fluid do not have any advantage over serum analysis. As the routine measurement of these markers in ascetic fluid is not easy in clinical practice and more invasive. These results were similar to a previous report by [8] who demonstrated a positive correlation between a group of tumor markers in serum and ascetic fluid in malignant ascites. On the contrary, [9] found that ascetic CEA was significantly higher than serum CEA in patients with advanced gastric cancer patients with ascites due to carcinomatosis.

Unpredictably, our results illustrated no statistical significant difference in the level of CEA messenger RNA between 3 groups. On the contrary, [10] indicated that quantification of CEA mRNA is useful for the evaluation of colorectal cancer progress as it is a predictive marker for micrometastasis. These conflicting results could be explained by the different technique.

The diagnostic work-up of ascites of unknown etiology (group I) included: ascetic fluid SAAG, cytology, abdominal US and CT scan, laparotomy with biopsy and other laboratory tests.

SAAG was $<1.1$ in $70.8 \%$ of patients with TB ascites and was $\geq 1.1$ in $71.4 \%$ of patients with cirrhotic ascites. This was comparable to reports $[11,12]$. Meanwhile, in ascites of unknown etiology SAAG was $<1.1$ in $73.2 \%$. the PPV of SAAG $<1.1$ in the diagnosis of non-portal hypertensive ascites was $89.5 \%$ comparable to [13], while, the NPV was $41.7 \%$ comparable to [11]. Ascetic fluid cytology revealed malignant cells in (9/41) $22 \%$ of patients with ascites of unknown etiology. When those undergone laparotomy and biopsy histopathology was positive for malignancy in 8 patients. Cytology had 53\% sensitivity, 94\% specificity, $89 \%$ PPV, and $70 \%$ NPV which was comparable to results by $[14,15]$.

On the other hand, our study showed that ascetic fluid cytology was negative for malignant cells in all TB ascites and cirrhotic ascites, including those whose abdominal US showed hepatic focal and confirmed by biopsy to be HCC. This was similar to a study by [16] who investigated the diagnostic yield of paracentesis in patients with cirrhosis, with or without HCC and found that cytology in cirrhotic patients, even those with known or suspected to have HCC, were almost always negative.

CT scan showed abnormalities in $66 \%$ of patients with ascites of unknown etiology in the form of: ovarian masses, colorectal lesions, hepatic focal lesions, and pancreatic lesions in $24.4 \%, 4.9 \%, 29.3 \%$, and $7.3 \%$ respectively [17].

Laparotomy diagnosed the etiology of 21/30 (70\%) of patients with ascites of unknown etiology who underwent laparotomy. Malignancy was found in 16/30 (53.3\%), of those, ovarian carcinoma, colorectal carcinoma, pancreatic carcinoma, HCC, hepatic heamangioma, metastatic carcinoma in the liver and peritoneal carcinomatosis were diagnosed in $43.8 \%, 12.5 \%, 12.5 \%, 12.5 \%, 6.3 \%$, $6.3 \%$ and $6.3 \%$ respectively. On the other hand, peritoneal tuberculosis was diagnosed in 4/30 (13.3\%) of cases and pancreatitis in $1 / 30(3.3 \%)$. Compared to our results, [18] had studied patients with ascites of unknown origin by laparoscopy and found the diagnostic yield was $87.2 \%$. The confirmed diagnoses were peritoneal carcinomatosis in $60.5 \%$, TB peritonitis in $20.2 \%$, and cirrhosis in $5.4 \%$. It has been reported that the most common cancers usually associated with ascites are adenocarcinomas of the ovary, breast, colon, stomach and pancrease [19].

TB ascites was proved by biopsy in 4/30 (13.3\%) of patients with ascites of unknown etiology. Those cases were presented as omental mass with or without mesenteric lymph nodes, peritoneal nodules, and ovarian mass with or without omental enlargement by laparotomy. These features may be noted also in patients with peritoneal carcinomatosis [20]. Therefore, patient may undergo unnecessary extended radical operation such as total hysterectomy, bilateral salpingo-oophorectomy, omentectomy and bilateral pelvic lymphadenectomy [21]. Consequently, confirmatory diagnosis in such situation should be established by biopsy.

Finally in this study, two (4.9\%) patients with ascites of unknown etiology remained undiagnosed even after reviewing the history and conducting more laboratory and imaging tests. This was comparable to [22] who reported $12 / 176(6.8 \%)$ patients who remained undiagnosed even after laparoscopy. Of note, those patients were males with mean(SD) 52 (4.2) years and negative for all tumor markers that we tested and they are being asked to keep following up with our team.

In conclusion, the etiological diagnosis of ascites of unknown etiology remains difficult to establish. A diagnostic work-up including a combination of ascetic fluid SAAG, cytology, imaging, and laparotomy with biopsy could be helpful in the diagnosis of ascites of unknown etiology. Our results confirmed that cytology has excellent specificity but low sensitivity for the diagnosis of malignant ascites. We also believe that no single tumor marker either in serum or ascetic fluid seems to be accurate enough to be used in the routine workup of ascetic fluid diagnosis. However, a tumor marker panel may represent a helpful adjunct to cytology and imaging studies in order to rule in malignancy as a probable etiology, therefore guiding the selection of patients who might benefit from further invasive procedures as laparoscopy or laparotomy with biopsy.

\section{REFERENCES}

[1] Han, C.M., Lee, C.L., Huang, K.G., Chu, C.M., Lin, S.M., Wang, C.J., et al. (2008) Diagnostic laparoscopy in ascites of unknown origin: Chang Gung Memorial Hospi- 
tal 20-year experience. Chang Gung Medical Journal, 31, 378-383.

[2] Alexandrakis, M., Moschandrea, J., Koulocheri, S., Kouroumalis, E. and Eliopoulos, G. (2000) Discrimination between malignant and nonmalignant ascites using serum and Ascitic fluid proteins in a multivariate analysis model. Digestive Diseases and Sciences, 45, 500-508. doi:10.1023/A:1005437005811

[3] Chen, S.J., Wang, S.S., Lu, C.W., Chao, Y., Lee, F.Y., Lee, S.D., et al. (1994) Clinical value of tumour markers and serum-ascites albumin gradient in the diagnosis of malignancy-related ascites. Journal of Gastroenterology and Hepatology, 9, 396-400.

doi:10.1111/j.1440-1746.1994.tb01262.x

[4] Sari, R., Yildirim, B., Sevinc, A., Bahceci, F. and Hilmioglu, F. (2001) The importance of serum and ascites fluid alpha-fetoprotein, carcinoembryonic antigen, CA 19-9, and CA 15-3 levels in differential diagnosis of ascites etiology. Hepato-Gastroenterology, 48, 1616-1621.

[5] Engelhardt, R., Fetscher, S. and Otto, F. (2008) Tumor markers. In: Berger, D.P., Engelhardt, M., Hen $\beta$, H. and Mertelsmann, R., Eds., Concise Manual of Hematology and Oncology, Springer, Berlin, Heidelberg, New York, 811-816.

[6] Pissaia, A., Jr., Bernard, D., Scatton, O., Soubrane, O., Conti, F. and Calmus, Y. (2009) Significance of serum tumor markers carcinoembryonic antigen, CA 19-9, CA 125, and CA 15-3 in pre-orthotopic liver transplantation evaluation. Transplantation Proceedings, 41, 682-684. doi:10.1016/j.transproceed.2008.12.015

[7] Simsek, H., Savas, M.C., Kadayifci, A. and Tatar, G. (1997) Elevated serum CA 125 concentration in patients with tuberculous peritonitis: A case-control study. The American Journal of Gastroenterology, 92, 1174-1176.

[8] Tuzun, Y., Celik, Y., Bayan, K., Yilmaz, S., Dursun, M. and Canoruc, F. (2009) Correlation of tumour markers in ascitic fluid and serum: Are measurements of ascetic tumour markers a futile attempt? The Journal of International Medical Research, 37, 79-86.

[9] Jung, M., Jeung, H.C., Lee, S.S., Park, J.Y., Hong, S., Lee, S.H., et al. (2010) The clinical significance of ascitic fluid CEA in advanced gastric cancer with ascites. Journal of Cancer Research and Clinical Oncology, 36, 517526. doi:10.1007/s00432-009-0684-3

[10] Miura, M., Ichikawa, Y., Tanaka, K., Kamiyama, M., Hamaguchi, Y., Ishikawa, T., et al. (2003) Real-time PCR (TaqMan PCR) quantification of carcinoembryonic antigen (CEA) mRNA in the peripheral blood of colorectal cancer patients. Anticancer Research, 23, 12711276.

[11] Das, B.B., Purohit, A., Acharya, U. and Treskova, E. (2001) Serum-ascites albumin gradient: A predictor of esophageal varices with ascites. Indian Journal of Pediatrics, 68, 511-514. doi:10.1007/BF02723242

[12] Khan, J., Pikkarainen, P., Karvonen, A.L., Mäkelä, T.,
Peräaho, M., Pehkonen, E., et al. (2009) Ascites: Aetiology, mortality and the prevalence of spontaneous bacterial peritonitis. Scandinavian Journal of Gastroenterology, 44, 970-974. doi:10.1080/00365520902964739

[13] Tuzun, Y., Yilmaz, S., Dursun, M., Canoruc, F., Celik, Y., Cil, T., et al. (2009) How to increase the diagnostic value of malignancy-related ascites: Discriminative ability of the ascetic tumor markers. The Journal of International Medical Research, 37, 87-95.

[14] Motherby, H., Nadjari, B. and Friegel, P. (1999) Diagnostic accuracy of effusion cytology. Diagnostic Cytopathology, 20, 350-357. doi:10.1002/(SICI)1097-0339(199906)20:6<350::AID-D C5>3.0.CO;2-7

[15] Castaldo, G., Oriani, G., Cimino, L., Topa, M., Mostarda, I., Castellano, L., et al. (1994) Total discrimination of peritoneal malignant ascites from cirrhosis- and hepatocarcinoma-associated ascites by assays of ascetic cholesterol and lactate dehydrogenase. Clinical Chemistry, 40, 478-483.

[16] Thrall, M.J. and Giampoli, E.J. (2009) Routine review of ascites fluid from patients with cirrhosis or hepatocellular carcinoma is a low-yield procedure: An observational study. CytoJournal, 6, 16. doi:10.4103/1742-6413.54919

[17] Callen, P.W., Marks, W.M. and Filly, R.A. (1979) Computed tomography and ultrasonography in the evaluation of the retroperitoneum in patients with malignant ascites. Journal of Computer Assisted Tomography, 3, 581-584. doi:10.1097/00004728-197910000-00001

[18] Yoon, Y.J., Ahn, S.H., Park, J.Y., Chon, C.Y., Kim, D.Y., Park, Y.N. and Han, K.H. (2007) What is the role of diagnostic laparoscopy in a gastroenterology unit? Journal of Gastroenterology, 42, 881-886. doi:10.1007/s00535-007-2106-0

[19] Saif, M.W., Siddiqui, I.A. and Sohail, M.A. (2009) Management of ascites due to gastrointestinal malignancy. Annals of Saudi Medicine, 29, 369-377. doi:10.4103/0256-4947.55167

[20] Gulati, M.S., Sarma, D. and Paul, S.B. (1999) CT appearances in abdominal tuberculosis. A pictorial essay. Clinical Imaging, 23, 51-59. doi:10.1016/S0899-7071(98)00090-4

[21] Piura, B., Rabinovich, A., Leron, E., Yanai-Inbar, I. and Mazor, M. (2003) Peritoneal tuberculosis-An uncommon disease that may deceive the gynecologist. European Journal of Obstetrics \& Gynecology and Reproductive Biology, 110, 230-234. doi:10.1016/S0301-2115(03)00101-5

[22] Han, C.-M., Lee, C.-L., Huang, K.-G., Chu, C.-M., Lin, S.-M., Wang, C.-J., et al. (2008) Diagnostic laparoscopy in ascites of unknown origin: Chang Gung Memorial Hospital 20-year experience. Chang Gung Medical Journal, 31, 310-313. 


\section{ABBREVIATION}

CA: cancer antigen; AFP: alphafetoprotein; PSA: prostate specific antigen; CEA: carcinoembryonic antigen; SAAG: serum ascetic albumin gradient; AST: aspartate aminotr- ansferase; ALT: alanine aminotransferase; IPD: intradermal purified protein derivative; CT: computarised tomography. 